\title{
Diagnostic value of sperm DNA fragmentation and sperm high-magnification for predicting outcome of assisted reproduction treatment
}

\begin{abstract}
Gemma López $^{1}$, Rafael Lafuente ${ }^{1}$, Miguel A Checa ${ }^{1,2}$, Ramón Carreras ${ }^{2}$ and Mario Brassesco ${ }^{1}$
Over the last years, major improvements in the field of male infertility diagnosis have been achieved. The aim of this study was to determine the diagnostic usefulness of sperm DNA integrity and sperm vacuolisation for predicting outcome in infertile couples undergoing in vitro fertilisation (IVF) and intracytoplasmic sperm injection (ICSI) treatments. A cohort study from 152 infertile couples undergoing sperm DNA fragmentation and high-magnification tests prior to an assisted reproduction treatment was designed. We found that the most predictive cutoff for pregnancy was $25.5 \%$ of DNA fragmentation with a negative predictive value of $72.7 \%(P=0.02)$. For the degree of vacuolisation, the best predictor of pregnancy was $73.5 \%$ of vacuolated sperm grades III+IV with a negative predictive value of $39.4 \%(P=0.09)$, which was not statistically significant. In conclusion, sperm DNA fragmentation greater than $25.5 \%$ could be associated with higher probability of failure IVF treatment. Regarding the results of the sperm analysis at high magnification, they do not allow us to predict whether or not patients will become pregnant.
\end{abstract}

Asian Journal of Andrology (2013) 15, 790-794; doi:10.1038/aja.2013.81; published online 5 August 2013

Keywords: DNA fragmentation; high-magnification; IVF; vacuoles

\section{INTRODUCTION}

Infertility is a common condition affecting one in six couples of childbearing age. In approximately $40 \%$ of these cases, a male factor is involved. The common definition for male infertility is the presence of an alteration in concentration, motility and/or morphology in at least one sample of two sperm analyses, according to World Health Organization guidelines. ${ }^{1}$

Basic semen analysis may not provide all information to completely evaluate male fertility status. Consequently, several investigators have considered optimizing conventional routine methods to improve male infertility diagnoses. Over the last two decades, the main investigation areas have been focused on sperm function, morphology and nucleus assessment. Major advances in the field of male infertility have been achieved, and these advances have resulted in numerous techniques for evaluating sperm chromatin quality and DNA fragmentation.

Sperm DNA fragmentation is increasingly suggested as a promising fertility predictive factor. ${ }^{2-4}$ Although there are some studies with opposite results, most of the published works report the association between DNA damage and lower fertilisation, impaired embryo cleavage and higher miscarriage rates. ${ }^{5-7}$

Sperm morphology has also been recognized to have an impact on fertilisation in in vitro fertilisation (IVF) treatments. ${ }^{8,9}$ One specific sperm malformation, the presence of large nuclear vacuoles (LNV), was described by Bartoov et al. ${ }^{10}$ after the development of a method to assess detailed sperm morphology. This novel method is called motile sperm organelle morphology examination and allows the evaluation of motile spermatozoa in real-time at a magnification of up to $\times 6600$. Some authors have demonstrated that the selection of spermatozoa for intracytoplasmic sperm injection (ICSI) with normal head morphology and size at high magnification improves pregnancy outcome (increasing pregnancy rate and reducing early abortion), ${ }^{11-14}$ suggesting that vacuolisation reflects some underlying chromosomal DNA defects. ${ }^{15}$

This study was designed to assess the diagnostic usefulness of sperm DNA integrity and sperm vacuolisation for predicting outcome in infertile couples undergoing IVF and ICSI treatments.

\section{MATERIALS AND METHODS}

In this cohort study, only the first attempts at IVF/ICSI procedures with ejaculated sperm were included. We consider 152 couples who visited our clinic from February 2009 to January 2011 and expressed an interest in participating in an IVF-ICSI program as a treatment for their infertility. The average time seeking for pregnancy prior to come to our clinic in this group of patients was 19.36 \pm 12.41 months (mean \pm s.d.). Only women with FSH levels lower than $10 \mathrm{mUI} \mathrm{ml}^{-1}$ and sperm samples with a concentration of 5 million sperm $\mathrm{ml}^{-1}$ or more were included.

To avoid potential sources of bias, we excluded women over 42 years old and treatment cycles that resulted in a poor ovarian response ( $<3$ mature oocytes collected) or those involving epididymal, testicular and cryopreserved sperm samples. Male patients having varicocele,

${ }^{1}$ Centro de Infertilidad y Reproducción Humana (CIRH), E-08017 Barcelona, Spain and ${ }^{2}$ Department of Obstetrics and Gynecology, Hospital Universitari del Mar, Universitat Autónoma de Barcelona, E-08003 Barcelona, Spain

Correspondence: Dr G López (glopez@cirh.es)

Received: 23 November 2012; Revised: 25 February 2013; Accepted: 30 May 2013; Published online: 5 August 2013 
leukospermia or under pharmacological treatment were also excluded from the study.

Written informed consent for participation was obtained from all patients on the day of the first semen sample collection. The study was approved by the Ethics Committee of the private center of reproductive medicine.

\section{Semen collection and preparation}

Semen samples were collected by masturbation, usually after 2-5 days of abstinence. Each sample was allowed to liquefy for at least $20 \mathrm{~min}$ at $37^{\circ} \mathrm{C}$. Basic sperm parameters including sperm count, concentration, motility and morphology were evaluated according to World Health Organization guidelines. ${ }^{1}$ After the initial assessment, ejaculates were divided into two aliquots. An aliquot of each sample was used to assess sperm DNA damage, and spermatozoa from the second aliquot were morphologically analysed using high magnification.

\section{DNA fragmentation assay}

The assessment of DNA damage was measured using an improved version of the sperm chromatin dispersion test (Halosperm kit; Halotech DNA S.L., Madrid, Spain). Samples were prepared for analysis according to the protocol described by Fernandez et al. ${ }^{2}$

A staining step is required to evaluate the prepared slides. The samples were stained with Diff-Quik solution (Diff-Quik; Dade Behring, Newark, DE, USA), immersing each slide in Diff-Quik solution I (eosinophilic) and Diff-Quik solution II (basophilic) for $6 \mathrm{~min}$ each, allowed to dry at room temperature and mounted with DPX (Fluka Chemie AG, Bruchs, Switzerland) on a $22 \times 22$ cover slide.

Laboratory technicians scored at least 500 spermatozoa for each patient under the $\times 100$ objective of the bright-field microscope according to the patterns established by Fernandez et al. ${ }^{6}$ When spermatozoa conserved the integrity of DNA, a peripheral halo of DNA loops around a central core was observed. Spermatozoa with fragmented DNA produced very small halos or no halos at all.

\section{Sperm morphology assessment}

The morphological assessment was conducted on a Nomarski interferential Leica AM 6000 (Leica DMI 6000B; Leica, Solms, Germany) inverted microscope equipped with a variable zoom lens. An aliquot of $1-2 \mu \mathrm{l}$ of the raw sperm sample was transferred to a microdroplet of polyvinyl pyrrolidone solution (PVP Clinical Grade; Origio Medicult Media, Malov, Denmark) and placed into a Petri dish (Willco-dish; Willco Wells BV, Amsterdam, The Netherlands) under sterile paraffin oil (Liquid paraffin; Origio Medicult Media).

The morphological assessment of the spermatozoa was performed as previously described by Vanderzwalmen et al. ${ }^{13}$ The spermatozoa were graded into four groups according to the presence and size of the vacuoles: grade I, no vacuole; grade II, a maximum of two small vacuoles $(<4 \%$ head surface); grade III, at least one large vacuole; and grade IV, a large vacuole and abnormal head shapes or other abnormalities.

To perform a correct sperm evaluation, each single sperm had to be followed by moving the microscope stage to observe the smallest details. Notably, motile sperm organelle morphology examination was applied exclusively to motile spermatozoa, which under low light microscopy magnification have a high potential to be selected for ICSI. One hundred motile spermatozoa from each sperm sample were examined microscopically at $\times 8000$ magnification.

To avoid variation in the interpretation of sperm magnification, all assessments were made by two specially trained laboratory technicians.

\section{Pregnancy outcome}

Clinical pregnancy was confirmed by the measurement of increasing concentrations of serum $\beta$-human chorionic gonadotropin on at least two occasions from day 12 after the embryo transfer and by the presence of at least one gestational sac with fetal heartbeat detection by transvaginal ultrasound examination.

\section{Statistical analysis}

Due to the lack of a reference for high-magnification diagnoses, the sample size was calculated to detect differences in DNA fragmentation between the subgroups of patients achieving pregnancy and those who did not. The pregnancy rate was estimated at $40 \%$; in accordance with our laboratory results and experience, for an alpha risk of 0.05 and a beta risk of 0.2 in a two-sided test, it was necessary to include 59 subjects in the pregnancy group and 88 patients in the non-pregnancy group to reach a statistically significant difference $\geqslant 5 \%$ of DNA fragmentation, with an assumed standard deviation (s.d.) of 10\%. A dropout rate of $10 \%$ was anticipated.

Differences between the groups of pregnancy and non-pregnancy were evaluated with the Mann-Whitney $U$ test for continuous variables. Receiver operating characteristic (ROC) curve analysis was performed to determine the prognostic accuracy of the sperm vacuolisation assessment and sperm DNA fragmentation as well as its ability to correctly classify subjects into successful and failed pregnancy subgroups. The threshold for each analysed parameter was calculated for optimal sensitivity and specificity. The positive predictive value, negative predictive value and their $95 \%$ confidence intervals (CIs) were also calculated for the cutoff point previously defined. Statistical significance was set to 0.05. Analysis was performed with SPSS 15.0 (SPSS Inc., Chicago, IL, USA).

\section{RESULTS}

Our sample size calculation indicated that 147 couples would provide enough power to detect statistically significant differences among groups. However, 152 couples interested in participating in the study were finally included. Table 1 reports baseline characteristics of the participants, divided into pregnant and non-pregnant couples.

With the ROC curve analyses, varying percentages of sperm DNA fragmentation and sperm vacuolisation values were used to calculate the optimum sensitivity and specificity regarding pregnancy outcome.

Table 1 The baseline characteristics of the study population $(n=152)$ and ART outcome. Results are expressed as mean \pm s.d.

\begin{tabular}{|c|c|c|c|}
\hline & Pregnant & Non-pregnant & P value \\
\hline Female's age (year) & $36.16 \pm 4.48$ & $36.86 \pm 4.19$ & 0.329 \\
\hline Male's age (year) & $37.09 \pm 4.75$ & $38.07 \pm 5.05$ & 0.219 \\
\hline \multicolumn{4}{|l|}{ Fresh sample } \\
\hline Count $\left(\times 10^{6} \mathrm{ml}^{-1}\right)$ & $64.02 \pm 59.41$ & $78.45 \pm 66.57$ & 0.190 \\
\hline Progressive motility (\%) & $43.02 \pm 23.45$ & $47.61 \pm 21.41$ & 0.244 \\
\hline DNA fragmentation (\%) & $18.90 \pm 7.71$ & $21.61 \pm 11.59$ & 0.102 \\
\hline $\begin{array}{l}\text { Vacuolisation grades } \\
\text { III+IV (\%) }\end{array}$ & $76.72 \pm 15.82$ & $76.21 \pm 13.79$ & 0.836 \\
\hline \multicolumn{4}{|l|}{ IVF-ICSI outcome } \\
\hline Oocytes retrieved & $11.63 \pm 5.79$ & $11.27 \pm 6.08$ & 0.517 \\
\hline Fertilisation rate $(\%)^{\mathrm{a}}$ & $71.99 \pm 17.10$ & $59.75 \pm 20.71$ & $<0.001$ \\
\hline Embryo rate $(\%)^{\mathrm{b}}$ & $70.77 \pm 17.98$ & $58.27 \pm 20.78$ & $<0.001$ \\
\hline
\end{tabular}

Abbreviations: ART, assisted reproduction treatment; ICSI, intracytoplasmic sperm injection; IVF, in vitro fertilisation.

${ }^{a}$ Fertilisation rate $=$ (No fertilized oocytes) $/($ No inseminated oocytes $) \times 100$.

${ }^{\mathrm{b}}$ Embryo rate $=($ No embryos $) /($ No inseminated oocytes $) \times 100$. 


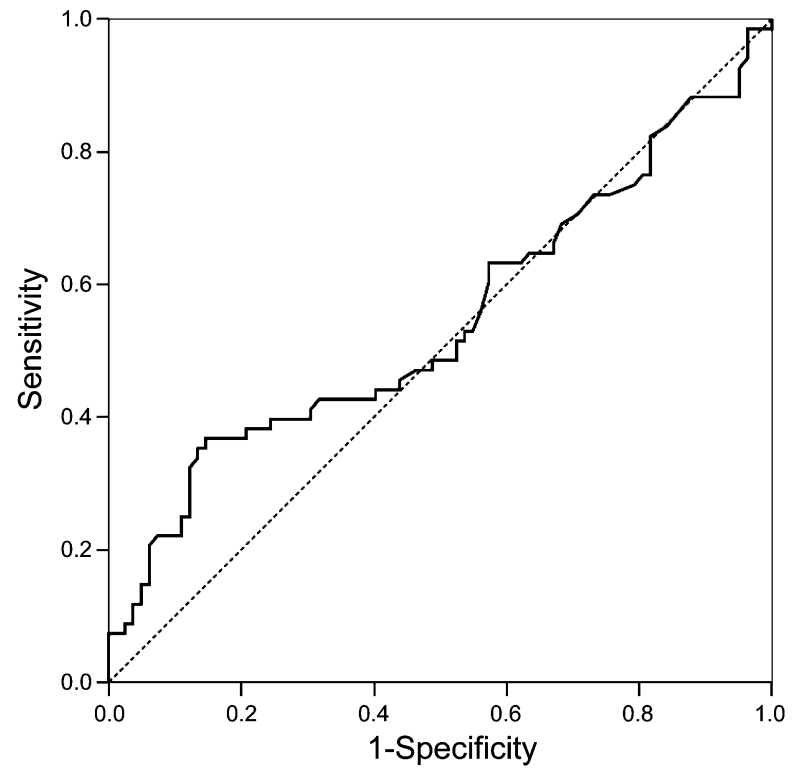

Figure 1 ROC curve for sperm DNA fragmentation. Area under the ROC curve (95\% Cl): 0.546 (0.450-0.642). ROC, receiver operating characteristic.

The best area under the ROC curve was 0.546 (95\% CI: 0.450-0.642) for $25.5 \%$ of sperm DNA fragmentation (Figure 1). The calculated threshold value for DNA fragmentation to distinguish between successful and unsuccessful IVF/ICSI treatments was $25.5 \%$. The odds ratio $(95 \% \mathrm{CI})$ for DNA damage to predict pregnancy is 3.60 (1.667.82) (Table 2).

For the analysis performed at high magnification in our male group, the best area under the ROC curve was 0.533 (95\% CI: $0.440-0.626$ ) (Figure 2). The predictive cutoff for pregnancy was observed when the sum of spermatozoa from grades III and IV was $73.5 \%$. The odds ratio (95\% CI) for sperm vacuolisation to predict pregnancy is $1.81(0.95-$ 3.44). (Table 3).

The multiple logistic regression shows that DNA fragmentation, sperm vacuolisation and number of embryos obtained per cycle are significantly independent variables related to pregnancies $(P=0.001$ 0.018 and 0.028 , respectively) with a good discrimination (area under the ROC curve $(95 \% \mathrm{CI}): 0.717(0.635-0.798))$ and calibration $(P=0.622)$ (Table 4).

Relationship between sperm DNA fragmentation and occurrence of large nuclear vacuoles, fertilisation rate and embryo rate was also calculated by Spearman's correlation test. A statistically significant correlation was found between sperm DNA fragmentation and sperm vacuolisation (Spearman's correlation $=0.275 ; P=0.001$ ). However,

Table 2 Prognostic accuracy of sperm DNA fragmentation to predict outcome after IVF/ICSI

\begin{tabular}{lccc}
\hline Statistical characteristics & Pregnant & Non-pregnant & P value \\
\hline DNA fragmentation (\%) & $18.90 \pm 7.71$ & $21.61 \pm 11.59$ & 0.102 \\
Area under ROC curve (95\% Cl) & $0.546(0.450-0.642)$ & \\
Cutoff (\%) & 25.5 & 0.029 \\
Sensitivity (\%) & 86.2 & \\
Specificity (\%) & 28.9 & \\
Positive predictive value (\%) & 48.7 & \\
Negative predictive value (\%) & 72.7 &
\end{tabular}

Abbreviations: ICSI, intracytoplasmic sperm injection; IVF, in vitro fertilisation; ROC, receiver operating characteristic.

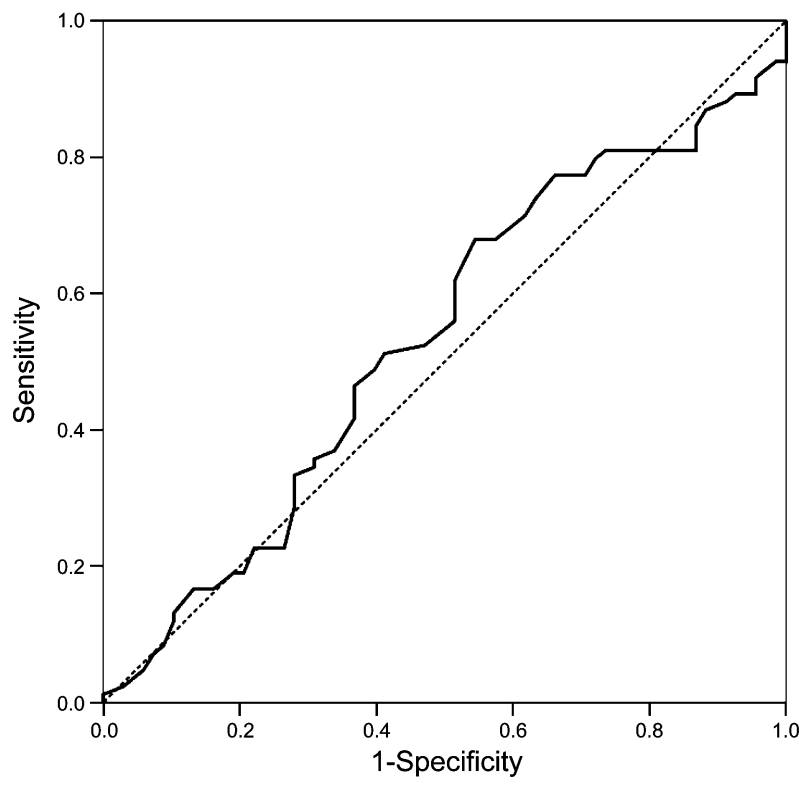

Figure 2 ROC curve for sperm vacuolisation grades III+IV. Area under the ROC curve $(95 \% \mathrm{Cl})$ : $0.533(0.440-0.626)$. ROC, receiver operating characteristic.

not statistically significant correlation was found between sperm DNA fragmentation and fertilisation and embryo rate (Spearman's correlation $=0.070 ; P=0.388 ;$ Spearman's correlation $=0.083 ; P=0.304$, respectively). Neither was between sperm vacuolisation and fertilisation and embryo rate (Spearman's correlation $=-0.018 ; P=0.817$; Spearman's correlation $=-0.010 ; P=0.901$, respectively).

\section{DISCUSSION}

In our study, the assessment of sperm DNA fragmentation was a better predictor of IVF/ICSI success than assessment of sperm using high magnification. There was no statistically significant relationship between the use of high magnification and success with IVF/ICSI.

Although several studies have shown the prognostic and diagnostic limitations of the routine semen parameters for the infertile couple, male infertility diagnosis is still based on the traditional semen analysis in routine clinical practice. ${ }^{7,16}$ However, these conventional semen parameters do not identify the subtle abnormalities in the male genome characterized by damaged sperm DNA. ${ }^{16-18}$

Sperm DNA damage is known to be associated with numerous indicators of reproductive outcome, including fertilisation, embryo quality, blastocyst formation, implantation and spontaneous miscarriage. $^{5,16,19,20}$ It has been shown that higher DNA fragmentation

Table 3 Prognostic accuracy of sperm vacuolisation grades III+IV to predict outcome after IVF/ICSI

\begin{tabular}{lccc}
\hline Statistical characteristics & Pregnant & Non-pregnant & P value \\
\hline Vacuolisation grades III-IV (\%) & $76.72 \pm 15.82$ & $76.21 \pm 13.79$ & 0.836 \\
Area under ROC curve (95\% CI) & $0.533(0.440-0.626)$ & \\
Cutoff (\%) & 73.5 & 0.090 \\
Sensitivity (\%) & 32.1 & \\
Specificity (\%) & 54.4 & \\
Positive predictive value (\%) & 46.6 & \\
Negative predictive value (\%) & 39.4 &
\end{tabular}

Abbreviations: ICSI, intracytoplasmic sperm injection; IVF, in vitro fertilisation; $\mathrm{ROC}$, receiver operating characteristic. 
Table 4 Multiple logistic regression for pregnancy outcome

\begin{tabular}{lcc}
\hline Variable & OR $(95 \%$ CI) & P value \\
\hline DNA fragmentation $(>25.5 \%)$ & $4.38(1.87-10.26)$ & 0.001 \\
Vacuolisation grades III+IV $(>73.5 \%)$ & $2.39(1.16-4.92)$ & 0.018 \\
Obtained embryos $(>4)$ & $2.39(1.10-5.19)$ & 0.028 \\
Female age & $0.940(0.87-1.02)$ & 0.121 \\
\hline
\end{tabular}

Abbreviations: $\mathrm{Cl}$, confidence interval; OR, odds ratio.

Discrimination power: AUC (95\% Cl): 0.717 (0.635-0.798).

Calibration of model: Hosmer and Lemeshow test.

indexes impair fecundity results. Larson et al. ${ }^{21}$ reported the absence of clinical pregnancy in patients with sperm DNA denaturation exceeding a threshold of $27 \%$ in the semen samples used in these IVF-ICSI cycles. Later studies indicate that DNA fragmentation levels above $30 \%$, as measured by the sperm chromatin structure assay, are associated with a probability of fertilisation at close to zero by intrauterine insemination. ${ }^{22}$

Although it is known that human spermatozoon has a highly dynamic and essential role in embryogenesis, the utility of sperm DNA testing is still a matter of debate. ${ }^{23-25}$ The evidence from current research is not strong enough to provide a clinical indication for the routine use of DNA damage assessment in infertility evaluation. ${ }^{4,16,26}$

In our study, the predictive value of sperm DNA fragmentation was assessed in a cohort of 152 ART couples using the sperm chromatin dispersion test. When the association between sperm DNA fragmentation and pregnancy was analysed, the results of the ROC curve analysis showed that DNA damage assessment was a good predictive parameter to distinguish between potentially pregnant and not potentially pregnant population. The cutoff point was at $25.5 \%$ sperm DNA fragmentation with a sensitivity of $86.2 \%$ and a specificity of $28.9 \%$. This threshold value for sperm DNA fragmentation in our study differs from the value suggested by the test manufacturer, which established a fragmentation index of $30 \% .^{6}$ In practice, when the sperm chromatin dispersion assay shows sperm DNA fragmentation index greater than or equal to $25.5 \%$ of the sample, we may expect an infertility rate of $72.7 \%$ for that patient.

The cause of sperm DNA fragmentation is still unclear. However, it is widely accepted that sperm quality and infertility are associated with chromatin remodelling defects during spermiogenesis and with oxidative stress. It has been demonstrated that the immune seminal cells, immature germ cells and mature sperm contribute to the production of reactive oxygen species that can cause DNA damage. ${ }^{16,27}$

The failure of sperm chromatin condensation has also been related to the presence of LNV by several authors. ${ }^{23,28,29}$ Franco et al. ${ }^{15}$ reported higher DNA fragmentation values in sperm nuclei with LNV. In a recent study, Perdrix et al. ${ }^{30}$ showed that sperm vacuoles were exclusively nuclear, and poor chromatin condensation and aneuploidy were the main alterations observed in spermatozoa with large vacuoles.

Assuming that nuclear vacuoles indicate chromatin abnormality, some investigators reported significantly lower pregnancy and implantation rates after transfer of embryos resulting from morphologically abnormal sperm cells. ${ }^{10,31,32}$

In our study, we decided to compare LNV (grades III+IV) sperm in reference to pregnancy rates because of the reported negative effect that LNV vacuoles exert on ICSI outcomes. ROC curve analyses were performed to determine the predictive value of high-magnification assessment in relation to pregnancy rates. Vacuolisation grades III+IV was not useful to discriminate between the groups of pregnant and non-pregnant women in IVF/ICSI cycles. The cutoff point was at $73.5 \%$, with a sensitivity of $32.1 \%$ and a specificity of $54.4 \%$.

In addition to DNA fragmentation, the origin and consequences of sperm head vacuoles are also a matter of controversy. According to Kacem et al., ${ }^{33}$ a large sperm head vacuole may originate from spermatogenesis impairment, abnormal maturation or modifications during the acrosome reaction. As a result, sperm defects may cause embryo developmental deficiencies resulting in clinical pregnancy failure or abortion. ${ }^{34,35}$

The present findings should be interpreted taking into account some limitations of the study, including the small study population, the advanced maternal age of the patients and the lack of stiffness of the inclusion criteria which may be a limiting factor to achieve higher pregnancy rates. As the policy of our laboratory is to perform half ICSI-half IVF in every cycle of treatment, we were not able to analyse separately these techniques. Moreover, high-magnification assessment by itself appears to be insufficient as a prognostic tool due to the later ICSI selection made at $\times 400$ with the conventional ICSI microscope. In spite of the several attempts to establish a standardized classification, there is a lack of consensus concerning the motile sperm organelle morphology examination (MSOME) and its classification. ${ }^{10,13,14}$ Of note, that to our knowledge, this is the first study in which highmagnification sperm morphology assessment is used as a diagnostic test rather than as a selection technique. Moreover, our results regarding sperm DNA fragmentation are in agreement with previous studies that reported a clear influence of DNA impairment on IVF/ICSI outcomes.

We conclude that sperm DNA fragmentation appears to be a useful technique to predict outcome in couples undergoing IVF/ICSI. Further studies are needed to confirm these encouraging preliminary findings as well to clarify the predictive potential of high-magnification sperm morphology for diagnostic purposes.

\section{AUTHOR CONTRIBUTIONS}

GL carried out the laboratory experiments, analysed the data, interpreted the results and wrote the paper. RL carried out the laboratory experiments, analysed the data, interpreted the results and revised the paper. MAC wrote the paper, revised the paper and analysed the data. RC revised the paper. MB designed the study and revised the paper.

\section{COMPETING FINANCIAL INTERESTS}

All authors declare no competing financial interests.

\section{ACKNOWLEDGMENTS}

The authors are grateful to Sergi Mojal, statistician, for his contribution to data analysis and Marta Pulido, MD, for editorial assistance.

1 World Health Organization. Laboratory Manual for the Examination of Human Semen and Sperm-Cervical Mucucs Interaction. 4th ed. Cambridge: Cambridge University Press; 1999.

2 Fernández JL, Muriel L, Goyanes V, Segrelles E, Gosálvez J et al. Simple determination of human sperm DNA fragmentation with an improved sperm chromatin dispersion test. Fertil Steril 2005; 84: 833-42.

3 Vélez de la Calle JF, Muller A, Walschaerts M, Clavere JL, Jiménez C et al. Sperm deoxyribonucleic acid fragmentation as assessed by the sperm chromatin dispersion test in assisted reproductive technology programs: results of a large prospective multicenter study. Fertil Steril 2008; 90: 1792-9.

4 Collins JA, Barnhart KT, Schlegel PN. Do sperm DNA integrity tests predict pregnancy with in vitro fertilization? Fertil Steril 2008; 89: 823-31.

5 Benchaib M, Braun V, Lornage J, Hadj S, Salle B et al. Sperm DNA fragmentation decreases the pregnancy rate in an assisted reproductive technique. Hum Reprod 2003; 18: 1023-8. 
6 Fernández JL, Muriel L, Rivero MT, Goyanes V, Vázquez R et al. The sperm chromatin dispersion test: a simple method for the determination of sperm DNA fragmentation. J Androl 2003; 24: 59-66.

7 Simon L, Brunborg G, Stevenson M, Lutton D, McManus J et al. Clinical significance of sperm DNA damage in assisted reproduction outcome. Hum Reprod 2010; 25: 1594608.

8 Ombelet W, Bosmans E, Janssen M, Cox A, Vlasselaer J et al. Semen parameters in a fertile versus subfertile population: a need for change in the interpretation of semen testing. Hum Reprod 1997; 12: 987-93.

9 Donnelly ET, Lewis SE, McNally JA, Thompson W. In vitro fertilization and pregnancy rates: the influence of sperm motility and morphology on IVF outcome. Fertil Steril 1998; 70: 305-14.

10 Bartoov B, Berkovitz A, Eltes F, Kogosowski A, Menezo Y et al. Real-time fine morphology of motile human sperm cells is associated with IVF-ICSI outcome. J Androl 2002; 23: 1-8.

11 Berkovitz A, Eltes F, Ellenbogen A, Peer S, Feldberg D et al. Does the presence of nuclear vacuoles in human sperm selected for ICSI affect pregnancy outcome? Hum Reprod 2006; 21: 1787-90.

12 Antinori M, Licata E, Dani G, Cerusico F, Versaci C et al. Intracytoplasmic morphologically selected sperm injection: a prospective randomized trial. Reprod Biomed Online 2008; 16: 835-41.

13 Vanderzwalmen $\mathrm{P}$, Hiemer A, Rubner $\mathrm{P}$, Bach $\mathrm{M}$, Neyer $\mathrm{A}$ et al. Blastocyst development after sperm selection at high magnification is associated with size and number of nuclear vacuoles. Reprod Biomed Online 2008; 17: 617-27.

14 Cassuto NG, Bouret D, Plouchart JM, Jellad S, Vanderzwalmen P et al. A new real-time morphology classification for human spermatozoa: a link for fertilization and improved embryo quality. Fertil Steril 2009; 92: 1616-25.

15 Franco JG Jr, Baruffi RL, Mauri AL, Petersen CG, Oliveira JB et al. Significance of large nuclear vacuoles in human spermatozoa: implications for ICSI. Reprod Biomed Online 2008; 17: 42-5.

16 Esteves SC, Agarwal A. Novel concepts in male infertility. Int Braz J Urol 2011; 37: 5-15.

17 Piasecka M, Gaczarzewicz D, Laszczyńska M. Evaluation of sperm genomic integrity of normozoospermic men: a prospective study. Folia Histochem Cytobiol 2006; 44: 117-22.

18 Miciński P, Pawlicki K, Wielgus E, Bochenek M, Gogol P et al. Total reactive antioxidant potential and DNA fragmentation index as fertility sperm parameters. Reprod Biol 2011; 11: 135-44.

19 Seli E, Gardner DK, Schoolcraft WB, Moffatt O, Sakkas D. Extent of nuclear DNA damage in ejaculated spermatozoa impacts on blastocyst development after in vitro fertilization. Fertil Steril 2004; 82: 378-83.

20 Larson-Cook KL, Brannian JD, Hansen KA, Kasperson KM, Aamold ET et al. Relationship between the outcomes of assisted reproductive techniques and sperm DNA fragmentation as measured by the sperm chromatin structure assay. Fertil Steril 2003; 80: 895-902.
21 Larson KL, DeJonge CJ, Barnes AM, Jost LK, Evenson DP. Sperm chromatin structure assay parameters as predictors of failed pregnancy following assisted reproductive techniques. Hum Reprod 2000; 15: 1717-22.

22 Bungum M, Humaidan P, Spano M, Jepson K, Bungum L et al. The predictive value of sperm chromatin structure assay (SCSA) parameters for the outcome of intrauterine insemination, IVF and ICSI. Hum Reprod 2004; 19: 1401-8.

23 Cassuto NG, Hazout A, Hammoud I, Balet R, Bouret D et al. Correlation between DNA defect and sperm-head morphology. Reprod Biomed Online 2012; 24: 211-8.

24 Álvarez JG. DNA fragmentation in human spermatozoa: significance in the diagnosis and treatment of infertility. Minerva Gineco 2003; 55: 233-9.

25 Sergerie M, Laforest G, Bujan L, Bissonnette F, Bleau G. Sperm DNA fragmentation: threshold value in male fertility. Hum Reprod 2005; 20: 3446-51.

26 Erenpreiss J, Spano M, Erenpreisa J, Bungum M, Giwercman A. Sperm chromatin structure and male fertility: biological and clinical aspects. Asian J Androl 2006; 8: $11-29$.

27 Muriel L, Meseguer M, Fernández JL, Álvarez J, Remohí J et al. Value of the sperm chromatin dispersion test in predicting pregnancy outcome in intrauterine insemination: a blind prospective study. Hum Reprod 2006; 21: 738-44.

28 Garolla A, Fortini D, Menegazzo M, de Toni L, Nicoletti V et al. High-power microscopy for selecting spermatozoa for ICSI by physiological status. Reprod Biomed Online 2008; 17: 610-6.

29 Oliveira JB, Petersen CG, Massaro FC, Baruffi RL, Mauri AL et al. Motile sperm organelle morphology examination (MSOME): intervariation study of normal sperm and sperm with large nuclear vacuoles. Reprod Biol Endocrinol 2010; 8: 56.

30 Perdrix A, Travers A, Chelli MH, Escalier D, Do Rego JL et al. Assessment of acrosome and nuclear abnormalities in human spermatozoa with large vacuoles. Hum Reprod 2011; 26: 47-58.

31 de Vos A, van de Velde H, Joris H, Verheyen G, Devroey P et al. Influence of individual sperm morphology on fertilization, embryo morphology, and pregnancy outcome of intracytoplasmic sperm injection. Fertil Steril 2003; 79: 42-8.

32 Bartoov B, Berkovitz A, Eltes F, Kogosovsky A, Yagoda A et al. Pregnancy rates are higher with intracytoplasmic morphologically selected sperm injection than with conventional intracytoplasmic injection. Fertil Steril 2003; 80: 1413-9.

33 Kacem O, Sifer C, Barraud-Lange V, Ducot B, de Ziegler D et al. Sperm nuclear vacuoles, as assessed by motile sperm organellar morphological examination, are mostly of acrosomal origin. Reprod Biomed Online 2010; 20: 132-7.

34 Barroso G, Valdespin C, Vega E, Kershenovich R, Avila R et al. Developmental sperm contributions: fertilization and beyond. Fertil Steril 2009; 92: 835-48.

35 Souza Setti A, Ferreira RC, Paes de Almeida Ferreira Braga D, de Cássia Sávio Figueira R, laconelli A Jr et al. Intracytoplasmic sperm injection outcome versus intracytoplasmic morphologically selected sperm injection outcome: a meta-analysis. Reprod Biomed Online 2010; 21: 450-5. 